DOI: https://doi.org/10.14232/actahisp.2021.26.27-41

\title{
MAÍZ, AGAVES Y ABEJAS COMUNES A MAYAS Y TEENEK
}

\author{
Jesús RuvalCaBa MERCADO
}

CIESAS Unidad Ciudad de México

\begin{abstract}
Resumen: La población teenek de la Huasteca y la maya de la península de Yucatán, ambas de la familia lingüística protomaya, ocupan los extremos opuestos del Golfo de México y a pesar de tantos cambios y problemas mantienen la producción del sistema agrícola de roza con el fin primordial de cultivar maíz y otros alimentos básicos como frijol, yuca, calabaza, chile y camote para el consumo familiar. Con todas sus similitudes también hay cuestiones que los diferencian. El objetivo no es compararlos en todos sus aspectos, sino solamente enmarcar y tratar de explicar cómo es que comparten una raza específica de maíz, la utilización de dos parientes cercanos de la familia agavaceae y el aprovechamiento variado de la miel de la abeja americana (melipona). Las referencias a otros temas como la organización del trabajo, los instrumentos agrícolas (la tecnología), su eficiencia, rendimientos agrícolas, medidas y usos conforman el contexto del problema. Los datos referentes a la Huasteca los obtuve durante el trabajo de campo y de la literatura existente, los referentes a los mayas provienen exclusivamente de otros autores que han escrito sobre el tema.
\end{abstract}

Palabras clave: agricultura milpera, auto abasto, organización familiar, familia protomaya.

Abstract: The Teenek people of the Huasteca and the Maya people of the Yucatán peninsula, both belonging to the Protomaya linguistic family, occupy opposite ends of the Gulf of Mexico and despite many changes and problems they maintain the production of slash-and-burn agricultural system with the primary purpose of growing maize and other staple foods such as beans, yucca, squash, chili and sweet potato for family consumption. Although all their similarities, there are also issues that differentiate them. The aim is not to compare them in all aspects, but only to frame and try to explain how they share a specific breed of maize, the use of two close relatives of the Agavaceae family and the varied use of the honey of the American bee (Melipona). References to other topics such as work organisation, agricultural implements (technology), their efficiency, agricultural yields, measurements and uses form the context of the problem. The data concerning the Huasteca were obtained during fieldwork and from existing literature while those concerning the Maya come exclusively from other authors who have written on the subject.

Keywords: Milpa Agriculture, Family Supply, Family Structure, Protomaya Family.

\section{Introducción}

La comparación productiva entre mayas y huastecos mayas (o teenek) me parece útil pero no suficiente de por sí. Es útil porque atrás de ella subyacen dos preguntas que la sacan de la mera curiosidad. Estas son: ¿qué nos pueden aportar las prácticas agrícolas acerca del parentesco conocido entre los mayas y los teenek? Y la que a mi juicio resulta más relevante: las similitudes y diferencias de sus sistemas productivos 
Maíz, agaves y abejas comunes a mayas y teenek

y aprovechamiento de los recursos a su alcance, ¿tienen que ver con su parentesco histórico o sólo son respuestas culturales en ambientes ecológicos parecidos? Vale recordar que ambos grupos pertenecen a la familia lingüística protomaya y que según estudios de la arqueología (Swadesh, 1953: 225) y de la glotocronología (Gruhn 1968) las dos lenguas y grupos se separaron hace alrededor de 30 siglos y que ahora se encuentran distanciados por casi $1500 \mathrm{~km}$ por la línea costera. Sólo como referencia, en el censo de 2020 se contabilizaron 780 mil hablantes de maya a nivel nacional, mientras que los teenek suman 168 729; es decir, ninguna se encuentra en el rango de riesgo de extinción.

En cuanto a la producción agropecuaria particular de ambos grupos, quedará fuera el uso de los huertos familiares o el aprovechamiento de los bosques, que son de suma importancia y requieren de un tratamiento aparte. Enseguida, sólo se destacarán tres elementos comunes a teenek y mayas peninsulares, de los que los grupos que se interpusieron entre ellos como totonacos, chontales, tepehuas y nahuas pueden compartir alguno, mas no el conjunto. Los tres componentes son el cultivo de una variedad de maíz, el designado como Tuxpan o tuxpeño cónico (Xnuc nal en maya - Ku Pech, 2019: 18) común a mayas peninsulares y teenek, el segundo es el cultivo y transformación de dos especies de los agaves textileros, el zapupe y el henequén, y, el tercero, el cuidado de abejas prehispánicas, conocidas por ambos pueblos y que, recientemente, ha cobrado verdadera importancia sobre todo por las propiedades curativas de su miel. El objetivo de estas líneas es tratar de encontrar una respuesta a través de tales procesos a las interrogantes enunciadas. Los datos de campo fueron recopilados en una estancia prolongada de 1980 a 1983 en dos localidades teenek, en otras estancias más cortas durante 2010 y 2012, y otros datos fueron obtenidos en visitas y recorridos por numerosas comunidades, la última a fines de 2018, para aplicar más de 500 encuestas a jefes de familia, hombres y mujeres nahuas y teenek de varias localidades.

\section{Las variables ecológicas}

La península de Yucatán en su mayor parte es una planicie calcárea de alrededor de $150000 \mathrm{~km}^{2}$ (si se suman los tres estados que la conforman: Yucatán, Campeche y Quintana Roo), más o menos uniforme en cuanto a su topografía. Por el contrario, la Huasteca (alrededor de $54000 \mathrm{~km}^{2}$ ) tiene tres zonas bien marcadas: la planicie costera, los lomeríos del pie de monte y la porción serrana con sus drásticos accidentes topográficos. Así que la vegetación de la región corresponde a cada uno de sus pisos ecológicos: en la planicie costera se asemeja a la de la Península de Yucatán, o sea, vegetación propia del trópico húmedo, la que impera en la Sierra Madre corresponde a grupos vegetales de transición entre la selva tropical y los bosques, y en las cimas impera el bosque de altura, compuesto en su mayoría de pinos y encinos, o lo que queda de ellos. De acuerdo con la capa arable 
y las lluvias, la península de Yucatán se dividía en cuatro grandes zonas: la que fue la zona henequenera al norte que es la más seca y pedregosa, la ganadera al suroeste, la hortícola y la maicera al oriente y sur de la península de Yucatán, con lluvias que permiten una cosecha de maíz al año y la ciudad de Valladolid como su centro urbano más importante. Esta distribución ha cambiado sobremanera en los últimos años de modo que ahora el cultivo del henequén ocupa sólo pequeñas franjas ( $\mathrm{Ku}$ Pech, 2019: 4, figura 1), mientras que han crecido desproporcionadamente las granjas avícolas y porcícolas con serios impactos sobre el ambiente. Por su parte, la Huasteca se subdivide en varias zonas agrícolas de acuerdo con la altitud: la zona ganadera que se ubica en la planicie costera, las zonas cañera y citrícola que se ubican en parte de la planicie y en los lomeríos de suaves ondulaciones, la franja maicera que ocupa nichos a veces planos, a veces con muy fuertes declives y la zona cafetalera en buena parte de los terrenos inclinados por arriba de los 700 u 800 metros sobre el nivel del mar. En suma, mientras que para la península yucateca el clima cambia en función de la latitud y la longitud, variables a las que para la agricultura se suman la precipitación anual, la cercanía con el océano y la profundidad del suelo agrícola, en la Huasteca las variables climáticas y, por lo tanto, la capacidad agrícola guardan una estrecha relación con la altitud sobre el nivel del mar además de la cercanía con el océano y las estibaciones de la sierra.

Una diferencia significativa es la forma en que corre el agua. En la Huasteca abundan arroyos y ríos superficiales (el Pánuco con sus numerosos afluentes es uno de los más voluminosos del país; además, cuenta con la laguna de Tamiahua), que pueden transformarse en torrentes intransitables durante la época de lluvias. En el paisaje serrano se multiplican las cascadas que adoptan alturas y formas caprichosas y llamativas. Por ejemplo, en el camino de Molango a Eloxochitlán, Hidalgo, por el camino de terracería que va por el bosque se pueden admirar una docena de ellas en un tramo de menos de $20 \mathrm{~km}$. Es en verdad un recorrido espectacular porque se suceden una tras otra, cada cual con su belleza, forma mudable y tamaño igualmente variable conforme cambia su caudal. En contraste, en la península los ríos son subterráneos con cenotes que también resultan espectaculares, ahora convertidos en atracciones turísticas. En las costas de ambas regiones se forman pantanos y manglares y, algo paradójico, es que dichas corrientes no se utilicen para el riego sino en muy poca proporción.

Dentro de la actividad agropecuaria de la Huasteca, en parte de la sierra, tanto la cantidad como la distribución de las lluvias durante el año permiten que los campesinos obtengan dos (y se han reportado tres) cosechas de maíz por año en la misma parcela, mientras que en la península de Yucatán sólo se cosecha una vez al año. En ambos casos, en la milpa, como se designa al longevo arreglo productivo, el maíz se asocia a varias otras especies como el frijol, el chile, el tomate, la calabaza, el camote, la yuca y, en Yucatán, con el macal y la jícama (Ku Pech, 2019: 1) además de frutales como el plátano y el chicozapote. Aunque el calendario agrícola anual es 
Maíz, agaves y abejas comunes a mayas y teenek

semejante (Mariaca Méndez, 2015: 13), otra distinción es la duración total del ciclo agrícola. En Yucatán la recuperación de la capa vegetal requiere alrededor de 12 o 15 años y, en contraparte, en la Huasteca se reduce a 6 o 12 años. Sabemos que al quemarla fertiliza todos los cultivos. Lo anterior es un factor decisivo en los sistemas agrícolas de roza, tumba y quema, descritos y evaluados por muchos investigadores alrededor del mundo. Por la abundante bibliografía al respecto, baste señalar que medir sus rendimientos totales, es decir, no sólo los del maíz (o del mantenimiento principal de que se trate) sino de todo lo que se produce en la milpa, en efecto resulta problemático cuantificarlos (Warman, 1985: 27) pero no imposible. Según el análisis pormenorizado del sistema en su conjunto como se practica en la Huasteca, los mismos igualan o superan el promedio de los sistemas mexicanos tecnificados, irrigados y fertilizados (Ruvalcaba Mercado, 2004).

Hoy día, en Yucatán como en otras partes del país, se registran de forma notoria parcelas no cultivadas. Esto se debe a varios factores como la migración, el prolongado periodo para que la tierra recupere la fertilidad, el acceso de los jóvenes a la educación y empleos en el sector de los servicios, los cambios climáticos, procesos de urbanización y los bajos precios de los productos agropecuarios. Otro riesgo latente es que la milpa - ese sistema considerado como un laboratorio biológico vivo en que se resguarda la sabiduría acumulada por siglos (Mariaca Méndez, 2015: 19)- y la diversidad de especies animales y vegetales seleccionadas a lo largo del tiempo también han sido afectadas para mal por diversas razones. Disminución de especies animales toleradas, razas de maíz, variedades de frijol, de tomate y otras especies asociadas, de los rendimientos decrecientes de todas ellas y de la superficie que año con año se sembraba son algunos de los problemas que enfrentan los campesinos huastecos y mayas. Otros factores de peso son el clima, la presión demográfica sobre la tierra, la suspensión del crédito y la edad de los cultivadores que por lo general rebasa los 50 años. Esto se torna grave porque se interrumpe la trasmisión a las nuevas generaciones de saberes y experiencias que tienen que ver con la milpa y también con otros conocimientos acumulados durante siglos, como hizo notar la Dra. Betty Faust sobre los depósitos u ollas subterráneas para captar y conservar agua de lluvia para la época de sequía, más otros conocimientos y sabiduría, dice ella, ampliamente antes compartidos pero que todavía se guardan en la memoria (2010: 175) Estas condiciones adversas no son recientes pues ya habían sido advertidas a fines del siglo pasado por varios estudiosos cuyo resumen analítico se puede consultar en Elena Lazos Chavero (1995) .

A pesar de lo anterior, el cultivo de la milpa se mantiene como un eje importante que articula varias otras actividades económicas y rituales de las familias mayas y teenek. Lo anterior tiene que ver con la necesidad de la familia de producir sus alimentos básicos, tanto como pueda, particularmente maíz. Se calcula que una familia campesina requiere de un almud de maíz por día, que equivale a entre 3,5 y 
4 kilos diarios o alrededor de 1500 kilos al año (Warman, 1985: 26). Lo medido para la Huasteca variaba según la época de trabajo y, obvio, el número de personas en la unidad. Para una familia de 3 adultos y 2 niños en temporada de trabajo el cálculo fue de más o menos 2 kilos de tortillas y medio kilo de frijol para la comida más fuerte. Es decir, alrededor de casi 3 kilos de maíz diarios y poco menos de un kilo de frijol más otros varios complementos y bebidas (Ruvalcaba Mercado, 1987).

\section{La organización del trabajo y los instrumentos agrícolas}

Es sabido que un sistema de roza requiere de abundante terreno agrícola y alrededor de 100 jornadas para producir maíz (Pérez del Ángel, 1993. Según diversos factores, Lazos Chavero -1995: 372- reporta entre 40 y 45 jornadas por hectárea para Yucatán), superficie necesaria puesto que a los años bajo cultivo los siguen otros tantos o más para que se recupere la cubierta vegetal, que en general se ve, de forma errónea, como un periodo de descanso. Lo anterior les hace ser considerados como sistemas agrícolas extensivos, cuando en no todos los casos es así. Si nos atenemos a los ejemplos etnográficos de la costa del Golfo, desde la Península hasta la Huasteca, la relación más común entre ambos periodos va de uno a uno hasta uno a 10. Es decir, desde un ciclo de cultivo por cada uno de recuperación, como el sistema marceño que, año con año, se practica en Tabasco con rendimientos de 5 o más toneladas por hectárea, de uno a dos como sucede entre los totonacos y en la Huasteca. En esa parte, se cultiva tres años seguidos y luego se deja la parcela por seis años en recuperación. En la franja maicera de Yucatán, la relación es de uno a diez, como en donde se cultivan dos o tres años seguidos y luego se deja la tierra hasta 20 años para que se recupere la cubierta vegetal. Allí se reporta el caso extremo (yo supongo que inusual también) de 50 años para que se recupere la cubierta vegetal (Hoil Gutiérrez, 2013: 99). No se requiere de un análisis más allá de la deducción para concluir que esta relación es una variable que depende mucho del clima. ${ }^{1}$ Los rendimientos promedio son de una tonelada de maíz por hectárea en la Huasteca para todo el ciclo (cultivo más descanso) y para la península de 400 a 600 kilos por hectárea por año, mientras que hace décadas se calculaban hasta 3 toneladas por hectárea (Lazos Chavero, 1995: 585).

La relación anterior se supone constante o casi en un ambiente donde hay tierra disponible en cantidades sobradas para sostener a la población existente, misma que puede alterarse no sólo por la densidad de población sino por cuestiones sociales

\footnotetext{
${ }^{1}$ Una comparación entre un sistema de roza, otro de año y vez con arado y otro de riego, su eficiencia, cantidad de tierra que requieren para su funcionamiento y la densidad de población en Palerm (1972a).
} 
Maíz, agaves y abejas comunes a mayas y teenek

como la tenencia de la tierra imperante, pero no se ha comprobado cuál de las dos variables, la ecológica o la estructural es la determinante. Incluso, varios autores sostienen que el colapso de la civilización maya durante el clásico se debió a la deforestación del medio. Sin embargo, si bien la deforestación, agotamiento y erosión están arqueológicamente documentadas (Abrams y Rue, 1988: 380), hay que suponer que la intensificación en el uso de las parcelas y, por lo tanto, el no dejar crecer la vegetación para recuperar la fertilidad de la capa arable, además de lo necesario para sostener una población densa, también se debió a las presiones del estado para extraer más excedentes de las familias campesinas. En otras palabras, al aumentar las presiones externas sobre la producción campesina, la unidad familiar reacciona con más hijos y con la intensificación de los sistemas agrícolas. Según Julio César Hoil Gutiérrez (2010: 50-51), algo semejante pasó con los mayas en la época colonial, cuando se vieron obligados a cubrir mayores demandas que les exigían los encomenderos.

Para explicar un poco más el asunto, en el caso de la Huasteca se ha dicho que en el siglo XIX, en el momento de la parcelación de los terrenos comunales por causa de las Leyes de Reforma, las comunidades en donde se practicaba la agricultura de roza tendieron a mantener una propiedad colectiva bajo la figura del condueñazgo, mientras que donde se cultivaba bajo otros sistemas tecnológicos, se aceptó sin tanto problema la adjudicación individual o familiar de las parcelas (Velasco Toro, 1989). Sin embargo, esta hipótesis no explica los ejemplos actuales porque en la Huasteca y, sobre todo, en Yucatán hay ejemplos de propiedades privadas o ejidales en donde se cultiva maíz con el sistema de roza. Por otro lado, en núcleos agrarios como son las congregaciones nahuas y teenek de la Huasteca la tierra está adjudicada en lotes familiares con límites fijos, en los que se practica también la roza. Lo anterior viene al caso puesto que en el ejemplo de la zona maicera de Yucatán, la mayor parte de la tierra está o estaba hasta hace poco en régimen ejidal. A pesar de ello, el cultivo de roza seguía con la antigua práctica necesaria de, junto con la milpa, trasladar familia y casa a la nueva parcela (Mariaca Mérndez, 2015: 10-11) o, bien, permitir acceso libre a los residentes del poblado para el cultivo de sus milpas mientras hubo tierra disponible (Torres-Mazuera Fernández Mendiburu, 2017: 169). Esto ha cambiado por varias razones como se indica más abajo.

\section{La eficiencia, rendimientos agrícolas, medidas y usos}

En ambos casos, en la milpa yucateca y en las de la Huasteca, el cultivo principal es el maíz, que se cultiva junto con otros alimentos básicos como diferentes variedades de frijol, calabaza, chile y otras hortalizas. La densidad de siembra es similar en los dos casos: alrededor de 50000 plantas de maíz por hectárea, con una distancia de alrededor de un metro entre cada golpe de cinco plantas de maíz en 
cada agujero y una distancia semejante entre hilera e hilera. La distribución vertical también es semejante: el maíz ocupa el estrato superior en cuyas cañas se enreda el frijol de guía, el de en medio lo ocupa el frijol de mata y las plantas de chile, y al estrato inferior lo cubren las plantas de calabaza que se extienden por la superficie de toda la parcela. Otras hortalizas y frutas se plantan en los linderos y varias de ellas, que nacen de forma silvestre, se cuidan o toleran como si fueran cultivadas.

Aquí hay un dato interesante que fue señalado por el Dr. Ángel Palerm y que se refiere a la raza de maíz cónico tuxpeño. Él señala que la discontinuidad de esa variedad de maíz (encontrada entre los mayas y los huastecos, pero no entre los totonacos) apunta la posibilidad de que fuera conocido por los dos primeros grupos desde antes de su separación a comienzos del Arcaico (Palerm, 1972).

$\mathrm{Ni}$ mayas ni huastecos domesticaron las primeras plantas de maíz, aunque en la Huasteca hay indicios muy antiguos de su presencia (MacNeish, 1955) y por lo visto de la intervención de sus pobladores para el mejoramiento y modificaciones ulteriores del grano y la mazorca. Lo anterior podría indicar que en los procesos de domesticación de esa planta y en los de la separación de la familia protomaya, todo el grupo cultivaba, junto con otras, esa variedad determinada de la que podrían reclamar la patente por su invención, porque es una de las razas más utilizadas para mejorar la especie por su facilidad de recombinarse y sus altos rendimientos. Es evidente que no se trata de una preferencia cultural ni de una adaptación puramente ecológica, puesto que totonacas, nahuas de Zongolica, ni los chontales de Tabasco la cultivan, todos ellos en ambientes si no iguales al menos muy parecidos. Se puede decir que esto es un indicio de la historia común, muy antigua, del grupo protomaya.

A pesar de lo anterior, también hay que decir que mayas y teenek cultivaban y cultivan otras variedades de maíz desde muy temprano (Mangelsdorf - MacNeish Galinat, 1967: 38-43) y, sobre todo, de los demás compañeros del maíz que su contraparte no conoce o no cultiva. Con los datos disponibles no hay forma de comprobar si, por ejemplo, la mayor cantidad de leguminosas que cultivan los mayas peninsulares a diferencia de las sólo dos o tres variedades de frijol (diferentes a las de sus parientes mayas) que cultivan los huastecos fueron domesticadas después de la separación de la gran familia, si se trata de préstamos por parte de los mayas peninsulares o de una pérdida en el repertorio agrícola antiguo de los huastecos. Otra diferencia es que si bien ambos sistemas, como todos los del mundo de este tipo, imitan la arquitectura vertical de la vegetación natural que substituyen, la riqueza de especies es mayor en la milpa yucateca. Como quedó advertido, lo anterior añade dificultades cuando se quieren hacer las mediciones del rendimiento total del ciclo completo.

El equipo tecnológico para el cultivo del maíz es similar por no decir igual: machete, hacha, coa, canastos y costales de ixtle para acarrear el producto, pizcadores de hueso o leznas para separar el totomoxtle de la mazorca y, de 
Maíz, agaves y abejas comunes a mayas y teenek

introducción reciente, desgranadoras hechas con los mismos olotes para desprender el grano del raquis u olote, aunque todavía se desgrana a mano. Las prácticas agrícolas son también semejantes aunque se practican y espacian una de otra de acuerdo a las condiciones climáticas en uno y otro lugar. El repertorio de instrumentos agrícolas es más amplio entre los mayas (Mariaca Méndez, 2015: 13), parece ser porque es el necesario para suelos pedregosos, pero no creo que sea el único motivo como se verá enseguida.

En cuanto a la organización del trabajo, en ambos casos se tiene como primordial la mano de obra familiar, a la que se suma otro tanto o más, la contratada sea por salario, sea por mano vuelta o ayuda recíproca -ahora casi en desuso total entre los mayas-, en ciertas labores agrícolas como son el desmonte, la siembra, las escardas y, a veces, la cosecha. En este rubro es notorio que la ayuda recíproca se ha perdido en mayor medida entre los mayas ${ }^{2}$. En la Huasteca, no sólo entre los teenek sino entre todos los otros pueblos originarios, la mano vuelta es una forma vigente y con mucha fuerza todavía. En esta también existe el trabajo colectivo, faena o fajina, que en muchas comunidades se utiliza para cultivar maíz en la o las parcelas colectivas aparte de los usos conocidos de limpiar caminos, construcción de edificios públicos, obra pública y limpiar pozos y escuelas las mujeres. En pocos de los ejidos yucatecos permanece esta tradición (Dr. Jesús Lizama, comunicación personal).

\section{El zapupe y el henequén}

Otro elemento particular que sólo comparten los mayas peninsulares y los teenek es el cultivo, comercio y transformación del henequén y del zapupe, parientes vegetales muy cercanos (incluso, aunque provienen del mismo ancestro, tal vez por confusión, comparten el nombre científico: Agave fourcroydes Lem., aunque me parece más preciso dejar $A$. zapupe Trel. para el zapupe y $A$. rigida para el henequén, por la diferencia notoria en la flexibilidad de su fibra), cultivados ambos solo para obtener el ixtle. A diferencia de sus parientes pulqueros, estas especies no suministran bebidas y ahora se sabe que fueron domesticados y ya se utilizaban antes de nuestra era. Según restos arqueológicos, la obtención de fibra de diversas especies de agave data de alrededor de 3 mil años antes de Cristo (Gentry, 1982, citado en Reyes Betanzos, 2009: 13-14). Aunque no se menciona en la Doctrina Christiana de Juan de la Cruz (1571) ni en un manuscrito de principios del siglo

\footnotetext{
2 Lo anterior se hizo evidente en los resultados del proyecto "Reconfiguraciones fundamentales de las sociedades originarias de México como efecto de la aplicación de políticas del Estado. Siglos XVI-XX”, llevado a cabo entre 2017 y 2020, en Yucatán, Oaxaca y la Huasteca. Quien firma coordinó los trabajos en esta última región.
} 
XVIII a pesar de que en este sí se hacen referencias al maíz y a la milpa (Hurch y Meléndez Guadarrama, 2020: 275). Tapia Zenteno en su vocabulario (1767) traduce zapup, que es la palabra en teenek, como pita (otro nombre para los magueyes y para su fibra), pero no vuelve a mencionarlo ni habla de cómo lo utilizaban. En una escultura prehispánica (De la Fuente - Gutiérrez Solana, 1980: 97, ilustración CCXXXIIIb) que representa a uno de los viejos asociados con la agricultura, parece claro que lleva un morral de ixtle, como hacen todavía hoy los huastecos al momento de la siembra.

El hecho de que hora los grupos asentados entre mayas y huastecos no cultiven el zapupe o el henequén llama la atención porque nahuas, otomíes y demás grupos indígenas de la Huasteca conocen la planta, usan de manera generalizada sus productos, pero no la cultivan ni elaboran mercancías o prendas a partir de sus fibras o lo hacen de manera muy esporádica. Son las comunidades teenek, las de San Luis Potosí y, sobre todo, las ubicadas alrededor de Tantoyuca, quienes se ocupan del zapupe y su transformación y, además, surten la demanda de sogas, cordeles y morrales. El morral huasteco se usa por casi toda la población -adultos y jóvenes, mujeres y niños- india y mestiza de la región, como antes de su mecanización e industrialización se hizo con el henequén. En la actualidad esta es sin duda la más grande diferencia: mientras que el henequén ha sido o fue industrializado casi por completo en las enormes haciendas henequeneras (asunto sobre el cual hay una abundante bibliografía), el zapupe se procesa con una tecnología al alcance de cualquier familia, hecha con los recursos del entorno, los instrumentos los fabrican los/las mismos/as tejedores/as aunque la elaboración de las múltiples prendas que se hacen con sus fibras requiere de una serie de conocimientos complejos que no cualquiera tiene y para lo cual se necesita de un largo entrenamiento. Llama la atención otro contraste: así como sobre el henequén los estudios de índole diversa sobrepasan la centena, para el caso del zapupe escasamente llegan a sumar una decena.

En el caso del henequén, si bien hay que señalar que es un planta domesticada por los mayas, en el siglo pasado ellos se vieron obligados a ser peones en las fincas que lo plantaban y en los lugares en donde se transformaba para su exportación. Cuando los hacendados incorporaron tecnología y maquinaria cara y sofisticada para procesar la fibra, los mayas perdieron también el control de su plantación, cultivo, transformación y comercio. A una pequeña característica entre la resistencia de cada una de las fibras se debe tanta diferencia en los procesos y sus resultados sociales. Mientras que la del henequén resiste la mecanización, la del zapupe no se pudo industrializar porque las máquinas desfibradoras rompen la fibra. De allí que los teenek conserven el control del proceso desde su plantación y cultivo hasta su transformación y comercio. Valdría la pena evaluar si ello influyó o cambió las condiciones materiales de vida de unos y otros, porque a simple vista no se notan tanto las diferencias: la mayoría de sus comunidades; unas y otras, siguen 
Maíz, agaves y abejas comunes a mayas y teenek

catalogadas como de alta o muy alta marginación. Sin embargo, es obvio que al menos en cuanto a evitar el despojo agrario, los teenek salieron ganando.

\section{Abejas prehispánicas y algunas propiedades curativas de su miel}

El tercer aspecto común es la obtención de miel de abejas prehispánicas (Melipona beecheii, domesticada) y otras especies silvestres, inofensivas porque no cuentan con aguijón, de las que se han documentado alrededor de 46 diferentes en México y 16 de ellas en Yucatán. Las zonas en donde se concentran son Centroamérica y la cuenca del Amazonas. A pesar de que antes y después de la conquista, mayas y teenek producían y se aprovechaban de la miel de unas y otras (Hoil, 2010: 120-123), hasta hace poco era más bien raro encontrar panales domesticados de estas abejas y también encontrar su producto en la Huasteca, aunque se usaba su miel para curar diversos males. Todavía esta miel no se utiliza tanto para endulzar sino por sus propiedades medicinales (Arellano, 1982: 73). De hecho, su rendimiento es muy menor (1,5 litro al año) comparado con el de la abeja europea (Apis melifera) que produce hasta 30 litros al año (Secretaría de Agricultura y Desarrollo Rural, 2018). No obstante, un gotero de $30 \mathrm{ml}$ de su miel cuesta hoy $\$ 250$, mientras que un frasco de 330 gr de miel de apis se cotiza hasta en $\$ 150$. Aunque sólo algunas familias teenek o mayas cuidan de estos colmenares de melipona, hoy día, a sus propiedades curativas probadas y a su precio mucho mayor que el de la miel común se debe que su promoción, cuidado y explotación se hayan expandido tanto en la península como por la Huasteca y otros lugares.

Entre los padecimientos y enfermedades que los "compuestos bioactivos tales como proteínas, flavonoides y polifenoles, con alta actividad antioxidante" de la miel melipona ayudan a combatir o mejorar se encuentran la cicatrización de heridas, pie diabético, fortalecimiento del nervio óptico y prevención de cataratas y algunos tipos de cáncer (Cauich Kumul - Ruiz Ruiz - Ortíz Vázquez - Segura Campos, 2015: 1432). Además se usa para trastornos del aparato digestivo, para aminorar los cólicos de la menstruación y numerosos usos dentro de la industria cosmética. Su miel tiene "propiedades antisépticas, antimicrobianas, anticancerígenas, anti-inflamatorias y de curación de heridas, proporcionando así defensas para las funciones celulares así como la promoción de las funciones celulares en los eritrocitos" (Cauich Kumul - Ruiz Ruiz - Ortíz Vázquez - Segura Campos, 2015: 1437).

En la actualidad se ha incrementado la demanda de la miel por empresas de diversos países dedicadas tanto a la salud como a los productos de belleza que se han enfrentado al problema de que la abeja melipona no prospera sino en ambientes y condiciones específicos, como los que imperan en la zona del Golfo de México. Por su lado, para los productores el problema es que el deterioro del hábitat donde viven estas abejas cada vez se agrava (Fernández Ruvalcaba, 2014), sin que reciban 
información para mejorar su cuidado y rendimiento ni estímulos fiscales $\mathrm{u}$ orientación para aumentar o facilitar a la vez las exigencias de calidad y homogeneidad y cumplir con los requisitos de exportación. Estas abejitas representan una alternativa para mejorar de forma significativa los ingresos de mayas, huastecos y totonacos ya que son ellos de quienes han tomado la información de los usos medicinales de su miel tanto los estudiosos, los laboratorios e incluso empresarios particulares.

En ambos lugares se produce también miel de la abeja europea (Apis melifera). La de Yucatán se cree de mejor calidad, así que casi toda la cantidad es para exportarla a Europa. Cada familia puede tener cien o doscientas colmenas, lo que representa un ingreso considerable. En años recientes, los productores pequeños y medianos enfrentan el grave problema de la siembra de cultivos genéticamente modificados, sobre todo soya por Monsanto, que -afirman- provoca daños al medio ambiente y la muerte de las abejas (Sánchez Osorio - Pardo Núñez - Flores López, 2018: 42). Otro endulzante se procesa a partir de la caña de azúcar de forma no mecanizada. Su jugo se extrae en trapiches movidos por animales, mismo que ya evaporado y cristalizado recibe varios nombres como piloncillo, chancaca, pilón o panocha. No obstante, no hay comparación en cuanto a lo que una y otra región produce: mientras la Huasteca en sus porciones de Veracruz y San Luis Potosí aportan casi el $30 \%$ del total nacional, Yucatán no figura entre los estados productores de caña y no cuenta con ningún ingenio (Secretaría de Agricultura y Desarrollo Rural, 2020).

Faltan por evaluar nuevas circunstancias como la vía del Tren Maya, el avance exponencial de la urbanización y de varias empresas en tierras antes ejidales o comunales, el abandono de la lengua originaria que, excepto el primero, son problemas comunes en una y otra región.

\section{Palabras finales}

Para terminar, una breve referencia a la comida de uno y otro lugar. Es indudable que hay diferencias notables a pesar de que el maíz, frijol y chile son los ingredientes básicos, esenciales, de una y otra región. Poco tiene que ver el zacahuil, típico tamal gigante de la Huasteca con los vaporcitos o tamales yucatecos. De la misma manera, los rellenos negro y blanco no tienen contraparte en la Huasteca. Quizá, lo único que comparten una y otra tradición culinaria sean las tortillas y los frijoles cocidos; de allí en adelante, la variedad es tanta que parecieran dos tradiciones totalmente distintas y distantes. Hay un par de atoles hechos con base en el maíz que se encuentran en ambas tradiciones. Ni siquiera los chiles, en extremo picosos que se comen por ambos pueblos de manera cotidiana son los mismos o parecidos; en la Huasteca se comen las variedades de $C$. annum mientras que en Yucatán se consume $C$. frutenses. Aprovecho para decir que si bien toda comparación tiene algo de veredicto, emitir un juicio sobre cuál tradición es más 
Maíz, agaves y abejas comunes a mayas y teenek

representativa, sabrosa o nutritiva no es la intención en este caso, ya que como dice el adagio "en colores y sabores no han escrito los autores". Baste decir que cualquier persona, por más exigente que sea, encontrará en uno y otro lado verdaderas delicias para su paladar y particularidades que sólo degustándolas (y no en cualquier lugar) se apreciarán por completo.

Como hemos visto, la historia y matriz cultural hacen de teenek y mayas parientes de la misma familia ecológica, lingüística y agrícola desde sus primeros pasos en la construcción de Mesoamérica. La abundante bibliografía existente sobre los mayas es incomparable con la que ha abordado el desarrollo o la actualidad de la Huasteca, también extensa pero lejos de la primera. Quedan muchos temas y procesos que habrían de investigarse de manera metódica y sistemática. En las líneas anteriores apenas se trató de llamar la atención en tres aspectos escasamente notados previamente. La mayoría de los estudios comparativos se han hecho con base en la grotocronología, la lingüística comparada, algunos rasgos arqueológicos y deidades o mitos compartidos. No obstante, en la creación cultural cada uno siguió caminos autónomos, independientes entre sí. Lo anterior nos indica sólo la antigüedad de su presencia en esa área cultural, pero nada nos dicen acerca de cómo se dio su separación. A mi parecer, apuntan a que toda la familia ocupaba la costa del Golfo y que la separación se debió a la intrusión de otros grupos que provenían del Altiplano o de regiones vecinas como Oaxaca. Sin embargo, como dije al principio, para esta llamada de atención esto es lo de menos. De mayor importancia son las implicaciones ecológicas con relación a las variables culturales. Si bien cada grupo ocupa un ambiente parecido, la respuesta cultural es una construcción que no tiene un solo camino. En este sentido, por ejemplo, hay mayores similitudes entre nahuas, otomíes y teenek de la Huasteca que entre los teenek y sus parientes mayas.

Estas similitudes se pueden ver en las formas de organizar el trabajo y también en la cosmovisión. No encuentro entre los mayas un personaje como Chicomexóchitl o Zipak que es un personaje ubicuo entre todos los grupos indios de la Huasteca, pero tampoco en la Huasteca hay un personaje como Chac. Faltan estudios sistemáticos entre ambas ramas de la familia que nos llevaría a saber, por ejemplo, si esas formas de gobierno prehispánico que en las crónicas se nos describen como pueblos sin señor universal tienen que ver con la forma en que concebían el poder los mayas y teenek, a una respuesta ecológica o a una etapa en la evolución del Estado Mesoamericano. Y así como esta debe haber muchas otras cuestiones por las cuales parece no sólo interesante sino de suma importancia emprender una comparación con tales objetivos. 
Jesús Ruvalcaba Mercado

\section{Agradecimiento}

Parte de los recursos provinieron del proyecto La Huasteca: Sociedad, Cultura y Recursos Naturales. Pasado y Presente, convocado por el CIESAS y El CONACYT (211100-5-G28649-H).

\section{Referencias bibliográficas}

Abrams, Elliot M. - Rue, David J. (1988). The Causes and Consequences of Deforestation among the Prehistoric Maya. Human Ecology, 16, 4. 377-395.

Arellano Tuz, Santiago (1982). El campesino maya y su economía a partir de la comunidad de Yalcobá, Yucatán. México: SEP-INI-CIESAS-Programa de Formación de Etnolingüistas.

Cauich Kumul, Roger - Ruiz Ruiz, Jorge Carlos - Ortíz Vázquez, Elizabeth Segura Campos, Maira Rubi (2015). Potencial antioxidante de la miel de Melipona beecheii y su relación con la salud: una revisión. Nutrición Hospitalaria, 32, 4. 14321442. Madrid: Grupo Aula Médica. Asequible en: https://www.redalyc.org/pdf/3092/309243319004.pdf, fecha de consulta 4 de febrero de 2021.

Cruz, Juan de la (1571). Doctrina Christiana en la lengua huasteca con la lengua castellana... México: Casa de Pedro Ocharte.

De la Fuente, Beatriz - Gutiérrez Solana, Nelly (1980). Escultura Huasteca en piedra. Catálogo. Cuadernos de Historia del Arte, 9. México: IIE-UNAM.

Faust, Betty Bernice (2010). El desarrollo rural en México y la serpiente emplumada. Tecnología y cosmología maya en la selva tropical de Campeche. Sección de Obras de Antropología. México: FCE/Cinvestav..

Fernández Ruvalcaba, Manuel (2014). Abejas nativas una oportunidad apícola en riesgo de extinción. Hypatia - Revista de Divulgación Cientifico - Tecnológica del Estado de Morelos. Asequible en http://hypatia.morelos.gob.mx, fecha de consulta: 1-03-2014.

Gruhn, Ruth (1968). A Lexic Statisting Dating of the Separation of Huastec and Chol. Antropologica, 10. 95-104.

Hoil Gutiérrez, Julio César (2010). El sistema milpero en el Yucatán colonial. Siglos XVIXVIII. Tesis de maestría en Historia. Mérida: CIESAS.

Hoil Gutiérrez, Julio César (2013). El sistema milpero de los mayas. Cambios y continuidades durante la época colonial. En: Lizama Quijano, Jesús J. (coord.). Entre irse y quedarse... Estructura agraria y migraciones internas en la Peninsula de Yucatán. México: Letra Antigua. 95-122. 
Maíz, agaves y abejas comunes a mayas y teenek

Hope, María Elena - Pereyra, Luz, eds. (1984). Nuestro maí. Treinta monografías populares. México: Museo Nacional de Culturas Populares-Consejo Nacional de Fomento Educativo-SEP.

Hurch, Bernhard - Meléndez Guadarrama, Lucero (2020). Conversación en lengua huasteca. Un manuscrito de las primeras décadas del siglo XVIII. Madrid: Iberoamericana Vervuert.

Ku Pech, Elia María (2019). La diversidad de maír, frijol y calabaza en la milpa maya de Xoy, Peto, Yucatán. Tesis de Maestría en Ciencias Biológicas. Mérida: Centro de Investigación Científica de Yucatán.

Lazos Chavero, Elena (1995). La milpa en el sur de Yucatán: dinámica y crisis. En: Hernández Xolocotzi, Efraim - Bello Baltazar, Eduardo - Levy Tacher, Samuel (eds.). La milpa en Yucatán. Un sistema de producción agrícola tradicional. Tomo 2. México: El Colegio de Posgraduados, 565-696.

MacNeish, Richard Stockton (1955). Ancient Maize and Mexico. Archaeology, 8. 108115. Asequible en www.jstor.org/stable/41663289, fecha de consulta: 14-03-2021.

Mangelsdorf, Paul C. - MacNeish, Richard S. - Galinat, Walton C. (1967). Prehistoric Maize, Teosinte, and Tripsacum from Tamaulipas, Mexico. Botanical Museum Leaflets, 22, 2. 33-63.

Mariaca Méndez, Ramón (2015). La milpa maya yucateca en el siglo XVI. Evidencias etnohistóricas y conjeturas. Etnobiología 13, 1: 25. Asequible en: https://www.revistaetnobiologia.mx/index.php/etno/article/view/71, fecha de consulta: 28-08-2021.

Palerm, Ángel (1972a). Aspectos agrícolas del desarrollo de la civilización prehispánica en Mesoamérica. En: Palerm, Ángel - Wolf, Eric (eds.). Agricultura y civilización en Mesoamérica. SepSetentas 32. México: SEP. 34-81.

Palerm, Ángel (1972b). Secuencia de la evolución cultural prehispánica de Mesoamérica: del arcaico a fines del clásico. En: Palerm, Ángel. Agricultura y sociedad en Mesoamérica. SepSetentas 55. México: SEP. 9-29.

Pérez del Ángel, Miguel (1993). El sistema de cultivo en Siloxúchil. En: Ruvalcaba, Jesús - Alcalá, Graciela (coords.). Huasteca II. Prácticas agrícolas y medicina tradicional. Arte y sociedad. Selección de trabajos pertenecientes al V y VI encuentros de investigadores de la Huasteca. México: CIESAS. 91-100.

Reyes Betanzos, Adlay (2009). Manejo del ixtle para la manufactura de productos útiles en la comunidad "teenek" de Xilozuchil, municipio de Tantoyuca, Veracruz. Tesis de licenciatura en Biología. Xalapa: Universidad Veracruzana.

Ruvalcaba Mercado, Jesús (1987). Vida cotidiana y consumo de maíz en el norte de Veracruz: Cuadernos de trabajo de la Casa Chata, 134. México: CIESAS.

Ruvalcaba Mercado, Jesús (2004). La agricultura de roza en la Huasteca indígena ¿suicidio o tesoro colectivo?. En: Ruvalcaba Mercado, Jesús - Pérez Zevallos, Juan 
Jesús Ruvalcaba Mercado

Manuel - Herrera, Octavio (coords.). La Huasteca, un recorrido por su diversidad. Colección Huasteca. México: CIESAS - El ColSan. 153-189.

Sánchez Osorio, Ever - Pardo Núñez, Joaliné - Flores López, María de Lourdes (2018). Conflictos ambientales en la producción apícola: resistencia maya ante la agricultura corporativa en la península yucateca. Tequio. Revista de Divulgación, Investigación e Innovación, 3, 1. 41-56. Oaxaca: UABJO.

Secretaría de Agricultura y Desarrollo Rural (2018). Melipona beecheii, "la abeja sagrada maya". Asequible en: https://www.gob.mx/agricultura/es/articulos/ melipona-beecheii-la-abeja-sagrada-maya, fecha de consulta: 21-08-2021.

Secretaría de Agricultura y Desarrollo Rural (2020). La caña de azúcar es el cultivo agrícola más importante del planeta. Y en nuestro país es una importante fuente de ingreso desde la época Colonial. Asequible en: https://www.gob.mx/agricultura/es /articulos/cana-de-azucar-una-dulce-produccion-237168, fecha de consulta: 21-082021.

Swadesh, Mauricio (1953). The Language of Archaeological Huastecs. Notes on Middle American Archaeology and Ethnology, 114. 223-227.

Tapia Zenteno, Carlos de (1767). Noticia de la lengua Huasteca. México: Imprenta de la Bibliotheca Mexicana.

Torres-Mazuera, Gabriela - Fernández Mendiburu, Jorge (2017). Los pobladores mayas de Chablekal versus el ejido de Chablekal. Una aproximación a la nueva demanda de tierra ejidal como territorio indígena de México. En: Bastos, Santiago Sierra Camacho, Teresa (coords.). Pueblos indígenas y Estado en México. La disputa por la justicia y los derechos. Colección México. México: CIESAS.

Velasco Toro, José (1989). La política desamortizadora y sus efectos en la región de Papantla, Veracruz. La Palabra y el Hombre, 72. 137-162.

Warman, Arturo (1985). Estrategia de sobrevivencia de los campesinos mayas. Cuadernos de Investigación Social 13. México: IIS-UNAM. 